\title{
Cost-effectiveness analysis of intensive blood-glucose control with metformin in overweight patients with Type II diabetes (UKPDS No. 51)
}

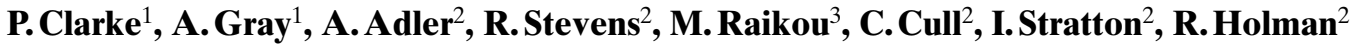 \\ on behalf of the UKPDS Group* \\ ${ }^{1}$ Health Economics Research Centre, Department of Public Health, University of Oxford, Institute of Health Sciences, \\ Headington, Oxford, UK \\ ${ }^{2}$ Diabetes Trials Unit, Nuffield Department of Clinical Medicine, University of Oxford, Oxford, UK \\ ${ }^{3}$ Department of Economics, City University, London, UK
}

\section{Abstract}

Aims/hypothesis. To estimate the economic efficiency of intensive blood-glucose control with metformin compared with conventional therapy primarily with diet in overweight patients with Type II (non-insulin-dependent) diabetes mellitus.

Methods. Cost-effectiveness analysis based on patient level data from a randomised clinical controlled trial involving 753 overweight ( $>120 \%$ ideal body weight) patients with newly diagnosed Type II diabetes conducted in 15 hospital-based clinics in England, Scotland and Northern Ireland as part of the UK Prospective Diabetes Study. Subjects were allocated at random to an intensive blood-glucose control policy with metformin $(n=342)$ or a conventional policy primarily with diet $(n=411)$. The analysis was based on the cost of health care resources associated with metformin and conventional therapy and the estimat- ed effectiveness in terms of life expectancy gained from within-trial effects.

Results. Intensive blood-glucose control with metformin produced a net saving of $£ 258$ per patient (1997 United Kingdom prices) over the trial period (median duration of 10.7 years) due to lower complication costs, and increased life expectancy by 0.4 years (costs and benefits discounted at $6 \%$ ).

Conclusions/interpretation. As metformin is both cost-saving in the United Kingdom and extends life expectancy when used as first line pharmacological therapy in overweight Type II diabetic patients, its use should be attractive to clinicians and health care managers alike. [Diabetologia (2001) 44: 298-304]

Keywords Economic evaluation, cost-effectiveness analysis, Type II diabetes, metformin, overweight patient, cost saving.
The United Kingdom Prospective Diabetes Study (UKPDS) has established that an intensive blood glucose control policy is effective in reducing the relative risk of any diabetes-related end point by $12 \%$ and of microvascular complications by $25 \%$ [1]. This land-

Received: 5 September 2000 and in revised form: 2 November 2000

Corresponding author: Dr. P. Clarke, Health Economics Research Centre, Department of Public Health, University of Oxford, Institute of Health Sciences, Headington, Oxford OX3 7LF, UK

Abbreviations: FPG, Fasting plasma glucose; QALY, qualityadjusted life years.

* see Acknowledgements mark study has shown also that the use of metformin for intensive blood glucose control in overweight patients confers a $32 \%$ risk reduction for any diabetesrelated end point and a $42 \%$ risk reduction for diabetes-related deaths compared with a conventional policy, primarily with diet alone. Metformin would seem to be advantageous as a primary pharmacological therapy in diet-treated overweight patients [2]. Although the cost-effectiveness of intensive blood glucose control has been ascertained [3], no study has so far examined prospectively the costs and benefits of using metformin. One study has reported that metformin can attain higher treatment success rates at lower cost than insulin therapy but this used an intermediate outcome measure $\left(\mathrm{HbA}_{1 \mathrm{c}}\right.$ value) and was based on data from a retrospective chart review of a 
Table 1. Main unit costs and sources

\begin{tabular}{lll}
\hline Item & Unit cost £s 1997 & Source \\
\hline UKPDS specialist clinic visit & $£ 67.30$ & Participating UKPDS centres \\
Metformin (500 mg/850 mg tablet) & $£ 0.02 / £ 0.03$ & BNF 1997 [11] \\
Other drugs & Cost per item & BNF 1997 [11] \\
In-patient day, in the 4 most frequently used specialties: & & \\
General surgery & $£ 271$ & Department of Health TFR2A costing returns 1996/ \\
Ophthalmology & $£ 689$ & 1997. Mean for English NHS Trusts, $n=$ up to 240 \\
Ear nose throat & $£ 511$ & \\
Cardiothoracic surgery & $£ 464$ & Average for 11 Trusts \\
Peritoneal dialysis (annual) & $£ 18140$ & DCCT [12], converted to £s 1997 by Purchasing Power \\
Retinal photocoagulation (course) & $£ 655$ & Parity \\
Other out-patient attendances & & Department of Health TFR2A costing returns 1996/ \\
General practitioner surgery visit & $£ 52$ & 1997, mean of English NHS Trusts \\
General practitioner home visit & $£ 10$ & Netten \& Dennett 1998 [9] \\
Diabetes specialist nurse visit & $£ 30$ & Netten and Dennett 1998 [9] \\
Practice nurse surgery visit & $£ 22$ & Netten and Dennett 1998 [9] \\
\hline
\end{tabular}

NHS, National Health Service; TFR, Trust financial returns; BNF, British National Formulary

small number of patients [4]. This paper reports an economic evaluation of metformin based on results from the UKPDS to establish whether the use of metformin is supportable on cost-effectiveness as well as clinical effectiveness grounds.

\section{Subjects and methods}

Patients, setting and comparison. The United Kingdom Prospective Diabetes Study has been described previously [1]. Briefly, between 1977 and 1991, 5102 patients with newly diagnosed Type II (non-insulin-dependent) diabetes mellitus aged 25-65 years and with a fasting plasma glucose greater than $6 \mathrm{mmol} / \mathrm{l}$ on two occasions were recruited to the study. After a dietary run-in period, metformin was included as a randomization option in overweight patients ( $>120 \%$ ideal bodyweight; body mass index approximately $25.6 \mathrm{~kg} / \mathrm{m}^{2} \mathrm{BMI}$ ) as part of the original protocol. In all, 342 overweight patients from the first 15 centres were allocated to an intensive blood-glucose control policy with metformin and 411 overweight patients were allocated to conventional treatment, primarily with diet alone. All patients continued to receive dietary advice at 3 monthly clinic visits with the aim of attaining normal bodyweight. In the conventional policy group the glycaemic goal was to obtain the lowest fasting plasma glucose (FPG) attainable with diet alone. In the intensive policy group the aim was a FPG of less than $6.0 \mathrm{mmol} /$ 1 by increasing the dose of metformin from 500 to $2550 \mathrm{mg}$ a day as required. If the FPG became greater than $15 \mathrm{mmol} / \mathrm{l}$ or hyperglycaemic symptoms developed on the maximum tolerated dose of metformin then glibenclamide was added. If pronounced hyperglycaemia recurred despite maximum sulphonylurea therapy, insulin was substituted for oral therapy [5].

Type of evaluation and perspective. The intention was to carry out a cost-effectiveness analysis in which the incremental net costs and net effectiveness (years of life gained) of metformin over conventional therapy were estimated and expressed as a ratio. Calculating the cost per life-year saved facilitates cost-ef- fectiveness comparisons with other life-prolonging interventions. Although quality of life could also be an important dimension of outcome, no reliable estimates of the utility associated with different diabetes-related health states are currently available, thus precluding the calculation of cost per quality-adjusted life-year. The perspective taken was that of the health care purchaser and so only direct health service costs were included in the analysis. These costs included treatment costs for metformin and conventional policies, visits to a nurse or general practitioner based on 'standard practice' assumptions (see below) and the costs of treating diabetic complications. All analyses and comparisons were done on an intention to treat basis.

Resource data. For each patient in the study, data were routinely collected on the dose of metformin as well as doses of all other drugs used for treating diabetes (insulin, sulphonylureas), of antihypertensive drugs (including captopril, atenolol) and the number of home blood glucose tests. Likewise whether the patient was taking aspirin, hormone replacement therapy, antidepressants, steroids or other drugs.

The date and duration of any hospital admissions were collected at each clinic visit. These were coded and classified by two clinicians as one of 40 national standard specialty codes [6]. For approximately $16 \%$ of admissions the length of stay was not recorded and so multiple imputation methods [7] were applied to replace the missing data. The variance was increased according to standard rules [8] to reflect the uncertainty surrounding the missing data. The costs associated with several expensive hospital stays that could significantly alter the results have been reported separately.

A cross-sectional survey of the use of all patients in the trials of non-inpatient healthcare resources was undertaken using a questionnaire distributed by the clinics between January 1996 and September 1997 and by post to those not attending a clinic during that period. Information was collected on all home, clinic and telephone contacts with general practitioners, nurses, podiatrists, opticians, dieticians and with eye and other hospital clinics over the previous 4 months. Each of these services was then assigned a unit cost based on published estimates [9] of health care costs (Table 1). The total cost, which 
Table 2. Annual visit and test schedules in a standard practice setting, judged to be equivalent to UKPDS levels of care, for intensive and conventional control policies, and resulting annual costs

\begin{tabular}{|c|c|c|c|c|}
\hline & \multicolumn{2}{|c|}{ Conventional } & \multicolumn{2}{|c|}{ Intensive with metformin } \\
\hline & Diet/tablet & Insulin & Diet/tablet & Insulin \\
\hline General practitioner nurse & 3 & 3 & 2 & 0 \\
\hline General practitioner clinic & 1 & 1 & 2 & 2 \\
\hline Physician at hospital diabetes clinic & 0 & 0.5 & 0.5 & 1 \\
\hline $\mathrm{HbA}_{1 \mathrm{c}}$ & 1 & 1 & 1 & 1 \\
\hline Home glucose tests per month & 1 & 11 & 1 & 11 \\
\hline
\end{tabular}

reflects resource use over the 4-month period covered by the questionnaire, was then multiplied by three to estimate each patient's total annual cost. A significant number of patients $(17 \%)$ recorded no non-inpatient costs over the survey period, making model specification difficult. For simplicity, a Tobit model [10] was used to estimate the relation between a patient's annual use of non-hospital resources and their age, sex, body mass index, duration of diabetes and recent history of non-fatal end points related to diabetes. The resulting equation was then used to estimate non-hospital resource use over the trial period. To check that results are not sensitive to this assumption, the analysis was repeated with a second model, derived from Poisson models for the numbers of visits in each non-inpatient category. The predicted number of visits was then multiplied by unit cost for each non-inpatient category. The effect of this alternative approach on the results is reported in the sensitivity analysis.

Costs. Table 1 summarises the main sources of information on unit costs, which were based primarily on United Kingdom national statistics and from centres participating in the trial. To obtain a cost per patient in the trial period unit costs were multiplied by resource volumes. Non-discounted costs are reported as well as net present values using the $6 \%$ annual discount rate approved by the United Kingdom Treasury; results using a $3 \%$ discount rate as recommended in the United States guidelines for health economic evaluations [13] are also reported. All costs are reported in 1997 values of pounds sterling.

All patients participating in the UKPDS, regardless of the policy to which they were allocated, attended clinics three or four times annually. As the type and frequency of visits is likely to be different in standard practice - especially for the conventional group - we report costs removing protocol-driven elements and using a pattern of clinic visits that reflects general practice and specialist clinical opinion on the implementation of a conventional or intensive policy (Table 2). A similar approach was adopted in previous economic analyses of the UKPDS [14] and the Diabetes Control and Complications Trial [12]. Each patient's standard practice costs were substituted for their actual trial visit costs when undertaking the analyses. The effects of alternative patterns of visits on the estimated costs are considered in sensitivity analyses.

Outcomes. We estimated the difference in life expectancy between patients allocated to metformin and to conventional policies. Where a patient was still alive at the end of followup, a simulation model described previously [14] was used to estimate the time from end of follow-up to death. Simulation models $[15,16]$ are often used in cost-effectiveness analyses of diabetic therapies because of the chronic nature of the disease. The estimated gain in life expectancy using our model is conservative because it assumes that beyond the trial period the two groups have identical hazard rates. A non-parametric bootstrap process, in which the metformin and conventional patients were sampled with replacement from the study cohort, was used to estimate uncertainty in the estimated results [17].

Analysis. All results are reported as mean values with standard deviations; mean differences are reported with $95 \%$ confidence intervals. To provide a visual representation of the results the costs and benefits are mapped onto the cost-effectiveness plane. Confidence intervals for the mean cost-effectiveness ratios were calculated using Fieller's method [18]. The effect of assumptions on our main results was examined using sensitivity analyses. All data were analysed using LIMDEP 7.0, SPSS 8.0 and Microsoft Excel 97 and the simulation model was written in $\mathrm{C}$.

\section{Results}

The mean (SD) age of patients was 53 (9) years and the mean body mass index $\left(\mathrm{kg} / \mathrm{m}^{2}\right)$ was $31.7(4.9)$. The median follow-up (to the last known date at which vital status was known or to the end of the trial) was 10.7 years.

Costs. Table 3 shows the mean cost per patient over the duration of the study by category of cost and by allocation.

The glucose control policy using metformin increased the total cost of therapy used in the intensive group by an average of $£ 1085$ (95\% CI. $£ 860$, $£ 1309)$ per patient compared with the conventional glucose control policy. This difference in costs was largely due to the greater number of visits to health professionals under the standard practice assumptions, with the costs of antidiabetic therapy being almost the same in both groups.

A large proportion of the costs of diabetes complications in the UKPDS can be attributed to hospital stays. The mean cost per patient of hospital stay in the conventional policy group was $£ 4632$ over the trial, compared with $£ 3317$ in the metformin group, a reduction of $£ 1315(-£ 2504,-£ 126)$. This difference arose primarily as a result of lower mean length of stay per hospital admission in the metformin group [mean of 9.6 days in the conventional group and 
Table 3. Mean costs over the follow-up period and mean cost differences, intensive policy with metformin compared with conventional policies, by cost category, £s 1997 (not discounted unless stated)

\begin{tabular}{|c|c|c|c|}
\hline \multirow[t]{2}{*}{ Item } & \multicolumn{2}{|c|}{ Mean (SD) cost per patient, $(£ s)$} & \multirow{2}{*}{$\begin{array}{l}\text { Mean cost difference } \\
(95 \% \text { CI }) \text {, per patient }{ }^{\mathrm{a}},(£ \mathrm{~s})\end{array}$} \\
\hline & Conventional & Metformin & \\
\hline Antihypertensive therapy & $716(963)$ & $701(950)$ & $-15(-152,122)$ \\
\hline Other drug therapies & $79(99)$ & $80(93)$ & $1(-13,15)$ \\
\hline Standard practice costs & $801(241)$ & $1808(536)$ & $1008(946,1069)$ \\
\hline Total therapy costs & $2157(1586)$ & $3242(1546)$ & $1085(860,1309)$ \\
\hline Hospital in-patient costs & $4632(10159)$ & $3317(6287)$ & $-1315(-2504,-126)$ \\
\hline Total complication costs & $6007(10391)$ & $4642(6551)$ & $-1365(-2589,-143)$ \\
\hline Total costs, standard practice & $8165(10904)$ & $7883(7086)$ & $-281(-1578,1015)$ \\
\hline $6 \%$ discount & $5893(7989)$ & $5635(4582)$ & $-258(-1171,655)$ \\
\hline $3 \%$ discount & $6878(9215)$ & $6607(5613)$ & $-271(-1345,801)$ \\
\hline
\end{tabular}

${ }^{a}$ Negative cost differences indicate cost savings associated with intensive blood glucose control policy using metformin. Sub-totals might not sum due to rounding

Table 4. Life expectancy and life years gained, from within-trial effect of treatment with metformin

\begin{tabular}{llll}
\hline Item & \multicolumn{2}{l}{ Mean (95\% CI) life expectancy (years) per patient } & \multicolumn{2}{c}{$\begin{array}{l}\text { Mean (95\% CI) life years } \\
\text { gained per patient }\end{array}$} \\
\cline { 2 - 4 } & Conventional & Metformin & $1.0(-0.0,2.1)$ \\
\hline Not discounted & $21.3(20.5,22.0)$ & $22.3(21.6,23.0)$ & $0.4(0.0,0.8)$ \\
3\% discount per year & $11.3(11.0,11.5)$ & $11.7(11.4,11.9)$ & $0.6(0.0,1.2)$ \\
\hline
\end{tabular}

8.0 days in the metformin group, a mean difference of 1.6 days $(95 \%$ confidence intervals $0.2,3.0)]$. The metformin group also had a lower mean number of hospital stays per patient but the difference was not statistically significant [1.6 in the conventional group, 1.4 in the metformin group, a mean difference of 0.2 $(-0.1,0.5)]$.

The analysis of non-hospital costs indicated that a recent clinical event increased costs on average by $£ 284$ in the first year, $£ 120$ in the second year and $£ 116$ in the third year after the event. The lower frequency of end points as a result of an intensive glucose control policy using metformin is therefore reflected in slightly lower non-inpatient costs over the course of the trial but the difference is not statistically significant.

The total costs associated with complications over the trial period were reduced by $£ 1365$ ( $-£ 2589$, $£ 143$ ) per patient in the metformin group, compared with conventional policy.

As the increased cost of therapy (including standard practice costs) amongst the metformin group is less than the reduction in the cost of complications, there is a net cost saving from the intervention. Discounted at $6 \%$ per annum to present values, the total cost of the conventional group is $£ 5893$ compared with $£ 5635$ in the metformin group. Hence an intensive glucose control policy with metformin produced an average cost saving of $£ 258(-. £ 1171, £ 655)$ per patient.
Three patients had very high cost episodes of care during the study that would have significantly altered the baseline results and are therefore reported separately. One patient on the conventional policy had two hospital episodes both exceeding 400 days for bipolar affective disorder. Including these costs increases the average hospital cost for the conventional group from $£ 4632$ to $£ 4932$ and thereby increases the overall undiscounted average cost difference between the conventional and metformin groups to $£ 582(-£ 2016, £ 853)$. One patient in each group received peritoneal dialysis during the study and if these costs are included the average cost difference between the conventional and metform groups are reduced to $£ 188(-£ 1500, £ 1125)$.

Outcomes. The main measure of effectiveness in this analysis is life-years gained. The mean modelled life expectancy was 21.3 years from the date of allocation in the group assigned to a conventional glucose control policy and 22.3 in the group assigned to an intensive glucose control policy with metformin, a mean difference of 1.0 (0.0 to 2.1) years. Discounted at a $6 \%$ rate, the difference in life expectancy was 0.4 (0.0 to 0.8$)$ years (Table 4$)$.

Cost-effectiveness. Figure 1 shows the cost-effectiveness plane, which simultaneously represents the difference in the mean costs (on the y-axis) and life expectancy (on the $\mathrm{x}$-axis) between the group treated in- 


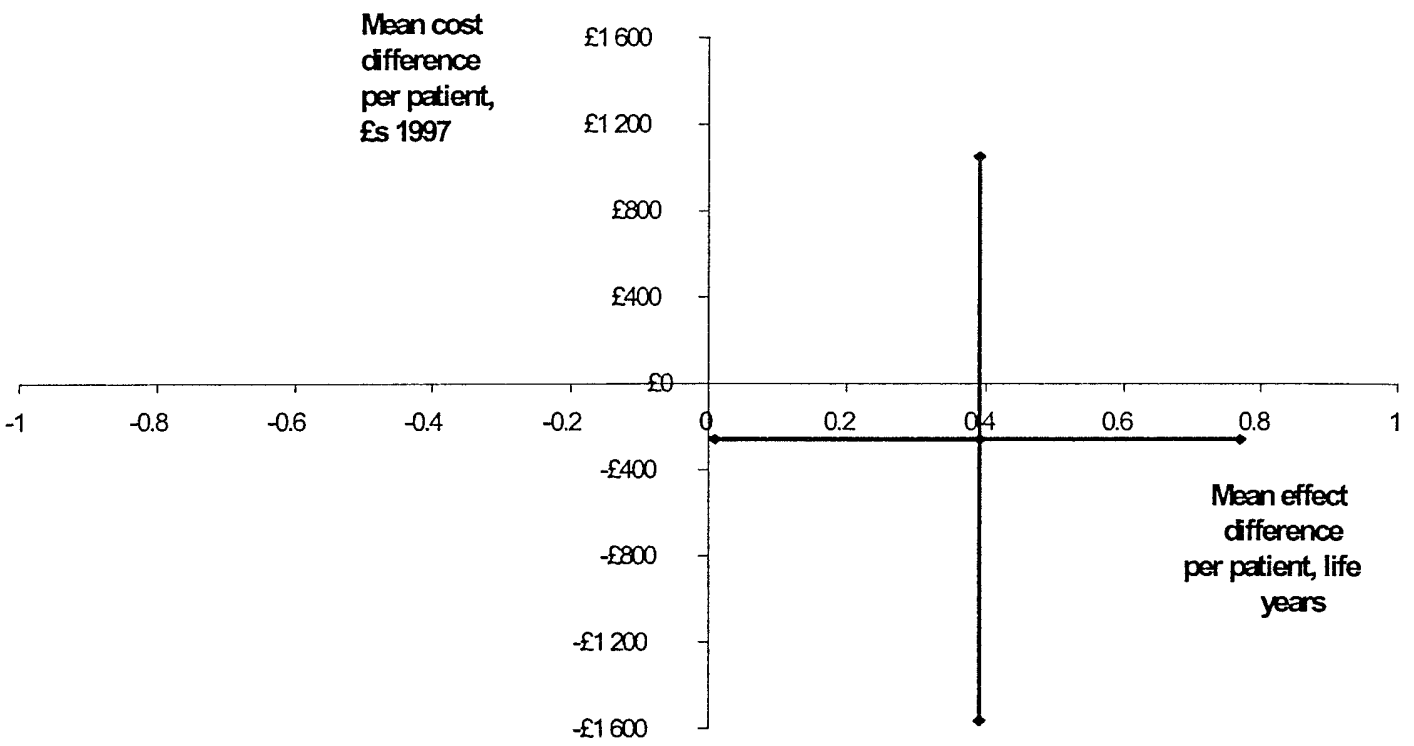

Fig. 1. Cost-effectiveness plane showing mean difference in cost and effect and $95 \%$ CI for metformin vs conventional therapy in overweight Type II diabetic patients

tensively with metformin and the conventional therapy group; both are shown discounted at $6 \%$ per year. The metformin policy group cost on average $£ 258$ ($£ 1171, £ 655)$ less than conventional policy and have a longer life expectancy of 0.4 (0.0 to 0.8$)$ years. Hence

Fig. 2. Cost-effectiveness acceptability curves: probability that the cost per life year gained is cost-effective (y-axis), as a function of the decision-maker's ceiling cost-effectiveness ratio (x-axis) intensive glucose control with a policy of metformin is both cost saving and more effective. In these circumstances, calculation of a cost-effectiveness ratio is not appropriate, as such a ratio would fail to differentiate between an intervention that was cost-saving and outcome enhancing, and an intervention that increased costs with poorer outcomes [19].

An alternative way of representing these results is in the form of a cost-effectiveness acceptability curve, which is shown in Fig. 2 [20]. The $\mathrm{x}$-axis shows a range of cost-effectiveness ratios from which decision makers can select a ceiling or maximum acceptable ratio and the $y$-axis shows the probability that the results are consistent with a true cost-effectiveness ratio being below that ceiling ratio. With costs and effects discounted at a $6 \%$ rate, there is a $95 \%$ probability that the cost-effectiveness of an intensive blood glucose

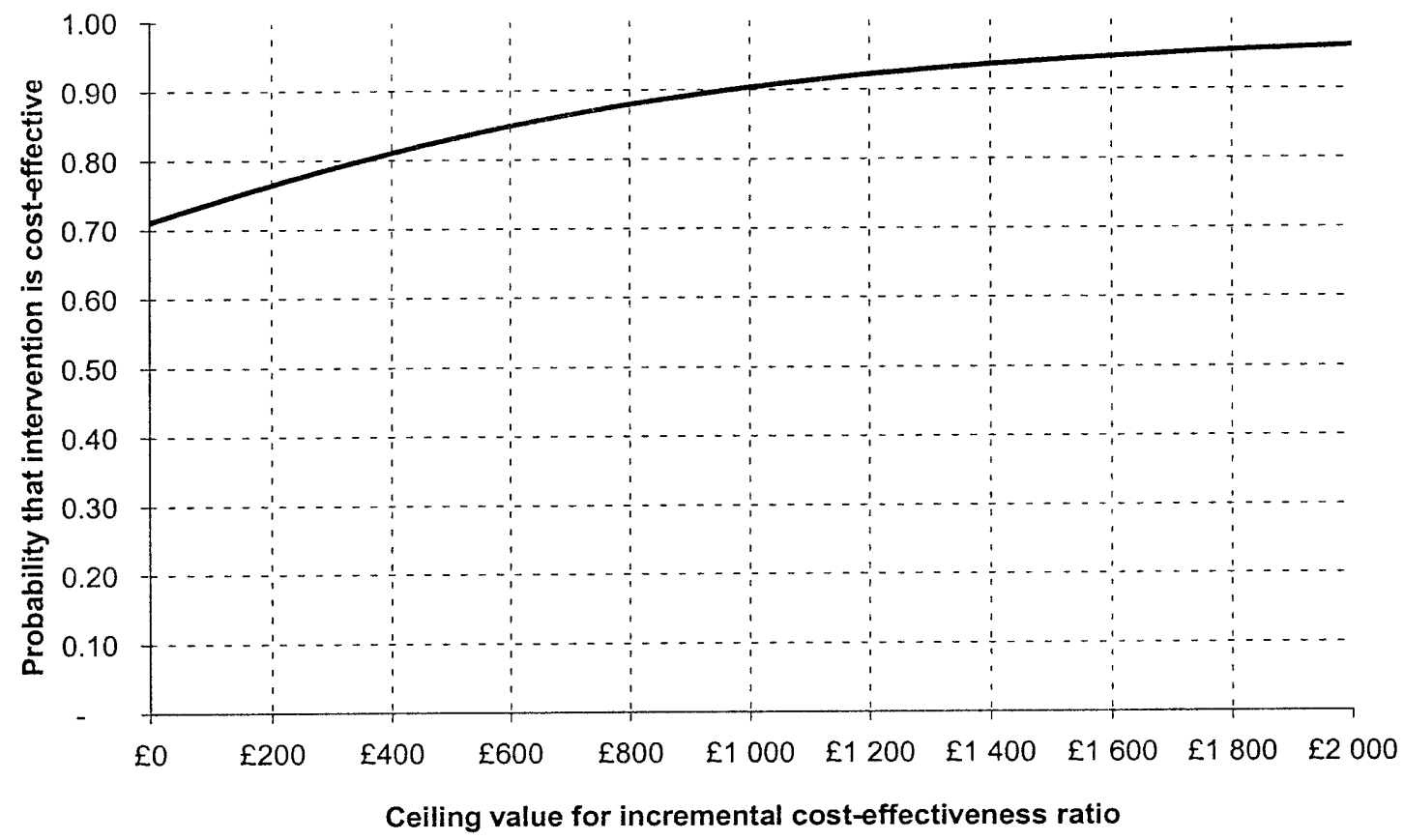


control policy with metformin is less than $£ 1600$ per life year gained and a $71 \%$ probability that metformin would prove to be cost-saving compared with a conventional policy.

Sensitivity analysis. Sensitivity analysis was done to examine whether the results in the main analysis are robust to different assumptions concerning metformin therapy in a standard practice setting, the cost of metformin and the modelling of non-inpatient costs. If the additional costs of intensive policy with metformin compared with conventional therapy were $50 \%$ more than assumed in the baseline estimates (see Table 2 for annual costs of standard practice), then intensive blood glucose control with metformin would be associated with a net cost of $£ 379$ ( $-£ 521$, $£ 1279$ ) per patient compared with a conventional policy. This would be equivalent to a cost per lifeyear gained of $£ 948$. Alternatively, if the additional costs of intensive policy with metformin compared with conventional therapy were $50 \%$ less than assumed in the baseline estimates, the reduction in costs between using a conventional policy and one of glucose treatment with metformin would become $£ 942(-£ 46,-£ 1839)$. If the cost of metformin was doubled then the reduction in costs between using a conventional policy and a policy of glucose treatment with metformin would become £106 (- £1022, $£ 809$ ). Finally, when a Poisson model is used to estimate non-inpatient costs an intensive glucose control policy with metformin is found to produce an average saving of $£ 269$ (-£1165, $£ 627$ ), showing that our results are robust.

\section{Discussion}

The results of this economic analysis of an intensive blood glucose control policy with metformin in overweight patients with Type II diabetes show that the reduction in the costs of treating the complications of diabetes offsets the additional costs of therapy. This results in an overall net saving.

We have previously shown that intensive blood glucose control with insulin or sulphonylureas is a cost effective means of increasing the time free of complications for patients with Type II diabetes [3]. In this analysis we have shown that intensive blood glucose control for overweight patients with metformin is a cost saving means of increasing life expectancy. These savings are largely due to the lower hospital in-patient costs incurred secondary to the major reduction in the risk of myocardial infarction for those patients on a policy of metformin [2]. Why metformin should have a greater effect in this regard than that attributable to the reduction in glycaemia [21] is not known although several mechanisms have been postulated $[2,22]$.
As the analysis has been done from the perspective of the health care system, it did not take into account losses of productivity to the patients and society. Because metformin was associated with shorter hospital stays, it is likely that metformin was also associated with fewer indirect and intangible costs, and this would accentuate the results reported here. The analyses also did not take into account some side effects such as abdominal discomfort and diarrhoea, which could have accounted for lower compliance with metformin compared with sulphonylurea or insulin [2]. The use of modest starting doses might increase compliance [23]. Nor did this study take into account that metformin was associated with fewer hypoglycaemic episodes and less weight gain than other therapies $[2,24,25]$. The costs associated with non-diabetic conditions for which high body weight is a risk factor should make metformin therapy even more attractive. Metformin requires no refrigeration and this could also produce cost savings in circumstances where refrigeration of insulin is an issue. Metformin has been shown to be an effective adjunct to insulin therapy in patients with Type II diabetes [26] and could provide practical advantages over insulin amongst the elderly [27].

The analyses we report use unit costs from the United Kingdom and these might differ from those in other countries. For example, the annual cost to the National Health Service of metformin at the median trial dose of $2550 \mathrm{mg} /$ day is $£ 43$ per patient, but until recently the cost of metformin treatment in the United States exceeded that of most other oral antidiabetic agents [22]. In our analyses even a doubling of the United Kingdom metformin cost was, however, associated with a cost saving.

Assumptions concerning standard practice also differ by country. In the United States, patients with diabetes in health maintenance organisations are typically seen more frequently ( 7 to 11 visits per annum [28]) than under the standard practice assumptions used here for the United Kingdom. Our sensitivity analyses indicate, however, that even with more frequent visits metformin would remain cost effective.

The observation that a large proportion of the observed total costs were attributed to hospital stays is consistent with previous estimates that they account for the overwhelming majority of costs in diabetes [29]. Patients treated with metformin did not have significantly fewer hospital admissions on average than the conventional treatment group but their stays were significantly shorter, at 8.0 days in the metformin group compared with 9.7 days in the conventional group. These lengths of stay for patients with diabetes are broadly comparable with data from the United States [29].

The very favourable cost-effectiveness of this therapy is not surprising given the clear prior evidence of effectiveness and the low cost of metformin in the 
United Kingdom. It should, however, be noted that medical interventions that are both cost saving and effective in prolonging life are comparatively rare. A recent review of published cost-effectiveness analyses in a wide range of therapeutic areas identified 60 studies that presented cost per life-year or cost per quality-adjusted life years (QALY) results in a United Kingdom context [30]. The median cost-effectiveness ratio of these studies was $£ 4961$ per life year/QALY gained. None of the studies reported that the interventions considered led to a net reduction in costs.

The analyses reported here evaluated metformin, initially as monotherapy, in overweight individuals with Type II diabetes (who make up the majority of patients studied in the trial). As metformin is both cost-saving and extends life expectancy, its use as the primary pharmacological therapy should be attractive to clinicians and health care managers alike.

Acknowledgements. The UK Prospective Diabetes Study (UKPDS) Group: Radcliffe Infirmary, Oxford; Royal Infirmary, Aberdeen; General Hospital, Birmingham; St George's Hospital and Hammersmith Hospital, London; City Hospital, Belfast; North Staffordshire Royal Infirmary, Stoke-on-Trent; Royal Victoria Hospital, Belfast; St Helier Hospital, Carshalton; Whittington Hospital, London; Norfolk \& Norwich Hospital; Lister Hospital, Stevenage; Ipswich Hospital; Ninewells Hospital, Dundee; Northampton Hospital. The co-operation of the patients and many National Health Service (NHS) and non-NHS staff at the centres is much appreciated. The major grants for this study were from the UK Medical Research Council, British Diabetic Association, the UK Department of Health, the National Eye Institute and the National Institute of Digestive, Diabetes and Kidney Disease in the National Institutes of Health, USA, the British Heart Foundation, NovoNordisk, Bayer, Bristol Myers Squibb, Hoechst, Lilly, Lipha and Farmitalia Carlo Erba. Other funding companies and agencies, the supervising committees, and all participating staff are listed in an earlier paper [1]. R. Stevens was supported by Wellcome Trust fellowship no. 054470/Z/98/Z/DG/NOS/fh. We are grateful to D. Groleau for providing research assistance, A. Briggs and D. Matthews for advice and P. Bassett for his assistance in the preparation of this manuscript.

\section{References}

1. UKPDS Group (1998) Intensive blood-glucose control with sulphonylureas or insulin compared with conventional treatment and risk of complications in patients with type 2 diabetes (UKPDS 33). UK Prospective Diabetes Study (UKPDS) Group. Lancet 352: 837-853

2. UKPDS Group (1998) Effect of intensive blood-glucose control with metformin on complications in overweight patients with type 2 diabetes (UKPDS 34). UK Prospective Diabetes Study (UKPDS) Group. Lancet 352: 854-865

3. UKPDS Group (2000) Cost effectiveness of an intensive blood glucose control policy in patients with type 2 diabetes: economic analysis alongside a randomised controlled trial. UKPDS 41. UK Prospective Diabetes Study Group. BMJ 320: 1373-1378

4. Brown RR (1998) Cost-effectiveness and clinical outcomes of metformin or insulin add-on therapy in adults with type 2 diabetes. Am J Health Syst Pharm 55: S24-S27

5. UKPDS Group (1995) United Kingdom Prospective Diabetes Study (UKPDS). 13: Relative efficacy of randomly allocated diet, sulphonylurea, insulin, or metformin in patients with newly diagnosed non-insulin dependent diabetes followed for three years. BMJ. 310: 83-88

6. Department of Health (2000) Hospital Episodes Statistics: Vol 1 , Finished consultant episodes by diagnosis, operation and specialty. England: Financial Year 1994-95. Department of Health, London

7. Schafer JL (1997) Analysis of Incomplete Multivariate Data. Chapman \& Hall, London

8. Little RJ, Rubin DB (1987) Statistical Analysis with Missing Data. John Wiley and Sons, New York

9. Netten A, Dennett J (1998) Unit costs of health and social care 1996. Personal Social Services Research Unit, University of Kent, Canterbury

10. Greene WH (1997) Econometric Analysis. Prentice-Hall, London

11. BNF (1997) British National Formulary. British Medical Association/Royal Pharmaceutical Society of Great Britain, London

12. DCCT (1996) Lifetime benefits and costs of intensive therapy as practiced in the diabetes control and complications trial. The Diabetes Control and Complications Trial Research Group. JAMA 276: $1409-1415$

13. Gold MR, Siegel JE, Russell LB, Weinstein MC (1996) Cost-effectiveness in health and medicine. Oxford University Press, New York

14. UKPDS Group (1998) Cost effectiveness analysis of improved blood pressure control in hypertensive patients with type 2 diabetes: UKPDS 40. UK Prospective Diabetes Study Group. BMJ 317: 720-726

15. Eastman RC, Javitt JC, Herman WH et al. (1997) Model of complications of NIDDM. I. Model construction and assumptions. Diabetes Care 20: 725-734

16. Palmer AJ, Weiss C, Sendi PP et al. (2000) The cost-effectiveness of different management strategies for Type I diabetes: A Swiss perspective. Diabetologia 43: 13-26

17. Carpenter A, Bithell J (2000) Bootstrap confidence intervals: when, which, what? A practical guide for medical statisticians. Stat Med 19: 1141-1164

18. Willan AR, O'Brien BJ (1996) Confidence intervals for cost-effectiveness ratios: an application of Fieller's theorem. Health Econ 5: 297-305

19. Stinnett AA, Mullahy J (1997) The negative side of cost-effectiveness analysis. JAMA 277: 1931-1932

20. Van HB, Al MJ, Gordon GS, Rutten FF (1994) Costs, effects and C/E-ratios alongside a clinical trial. Health Econ 3: 309-319

21. UKPDS Group (2000) Association of glycaemia with macrovascular and microvascular complications of type 2 diabetes (UKPDS 35): prospective observational study. BMJ 321: 405-412

22. DeFronzo RA (1999) Pharmacologic therapy for type 2 diabetes mellitus. Ann Intern Med 131: 281-303

23. Selby JV, Ettinger B, Swain BE, Brown JB (1999) First 20 months' experience with use of metformin for type 2 diabetes in a large health maintenance organization. Diabetes Care 22: 38-44

24. Johansen K (1999) Efficacy of metformin in the treatment of NIDDM. Meta-analysis. Diabetes Care 22: 33-37

25. Makimattila S, Nikkila K, Yki JH (1999) Causes of weight gain during insulin therapy with and without metformin in patients with Type II diabetes mellitus. Diabetologia 42: 406-412

26. Aviles SL, Sinding J, Raskin P (1999) Effects of metformin in patients with poorly controlled, insulin-treated type 2 diabetes mellitus. A randomized, double-blind, placebo-controlled trial. Ann Intern Med. 131: 182-188

27. Saudek CD, Hill GS (1999) Feasibility and outcomes of insulin therapy in elderly patients with diabetes mellitus. Drugs Aging 14: 375-385

28. Engelgau MM, Geiss LS, Manninen DL et al. (1998) Use of services by diabetes patients in managed care organizations. Development of a diabetes surveillance system. CDC Diabetes in Managed Care Work Group. Diabetes Care 21: 2062-2068

29. ADA, American Diabetes Association (1998) Economic consequences of diabetes mellitus in the U.S. in 1997. Diabetes Care 21: 296-309

30. Briggs A, Gray A (1999) Handling uncertainty when performing economic evaluation of health care interventions. Health Technol Assess 3: 1-134 